\title{
Clotting detection in the vascular network
}

\author{
Shatha A. Salman, Abeer H. Abd-Almeer \\ Mathematics and Computer Application, Applied Sciences, University of Technology, Iraq
}

\begin{tabular}{l}
\hline \hline Article Info \\
\hline Article history: \\
Received Feb 20, 2020 \\
Revised Apr 21, 2020 \\
Accepted May 5, 2020 \\
\hline Keywords: \\
Algorithm \\
Connected graph \\
Graph \\
Segmentation \\
Vascular network
\end{tabular}

\begin{abstract}
A lot of side effects accusers which the humans have suffered from a scan either by CT phrases, rays and magnetic resonance. Different methods were used to eliminate these affects depends on segmentation of vascular networks then represents it as a graph, where the intersection of the vessel as a vertex and the line between them as an edge. The place of the clot can be found by representing the weight of each edge as the amount of the blood in the vessel. Sign the place; if the amount is less than the normal flux that represents thrombosis. An algorithm in a graph theory is used to find the minimum distance, if more than one thrombosis exists to reach the nearest one and sign it alternately.
\end{abstract}

Copyright () 2020 Institute of Advanced Engineering and Science. All rights reserved.

Corresponding Author:

Shatha Assaad Salman, Department of Applied Sciences, University of Technology, Al-Sinaa Street, Baghadad, Iraq. Email: 100178@uotechnology.edu.iq

\section{INTRODUCTION}

Graph is construction that have a long history in science that applied in pretty much every logical and building, many picture division techniques use graph construction when speaking to picture [1-3]. By using mathematical methods, the solution of the problem in a picture is found in a simple and adaptable manner. The utilization of diagrams for a constructional illustration of thing models took out from images. Medical image analysis is the science that supports medical problem analysis completely different imaging methods and computerized image examination methods and systems [4, 5]. Segmentation is characterized as the methodology of isolated a picture into a lot of meeting areas that characterized for each homogeneous locale, where is change starting with one district then onto the next is sharp [6].

The base cost associated subgraph improvement issue appears in various therapeutic picture investigation undertakings, the chief evident is for portioning neural structures [7], reproducing tube systems [8], varieties of this headway issue have been suggest for anatomical naming of vaesculature [9], conduit vessel division [10]. For the preliminaries which identifies graph hypothesis refer to [11-13]. Let us now give way to enter the main work in the research. Let $(\mathrm{V}(\mathrm{G}), \mathrm{E}(\mathrm{G})$ ) be a graph $(\boldsymbol{G})$ where $\mathrm{V}(\mathrm{G})$ may be a nonempty set whose parts are referred to as points (vertices). E (G) may be a set of unordered pairs of elements of $V(G)$. The elements of $E(G)$ are called lines (edges) of the graph $G$. A graph $G=(V, E)$ with $n$ vertices and $\mathrm{m}$ edges is termed a (n, m)-graph. A graph with no multiple edges, no loop is said to be $\boldsymbol{a}$ simple graph. A graph $H$ may be a graph of $G$ if each vertex of $H$ may be a vertex of $G$, and each edge of $H$ is an edge of $G$. $H$ may be $\boldsymbol{a}$ spanning sub graph of $\mathrm{G}$ if $H$ includes all vertices of $G$. In [14] gives the way of reaching the clotted in less time but in this paper the location of the clotted vessels is signed. A succession of vertices without repetition of order $n$ is called a path graph $\left(\boldsymbol{P}_{\boldsymbol{m}}\right), m \geq 1$ and size-1, the vertices are labeled $V_{1}, V_{2}, \ldots, V_{m-1}, V_{m}$ and the edges are $V_{1} V_{2}, V_{2} V_{3}, \ldots, V_{m-1} V_{m}$ and if is closed is said to be a cycle graph $\left(\boldsymbol{C}_{m}\right)$ of order and size $m, m \geq 3$. A graph $G$ is alleged to be connected graph if each combine of 
vertices are joined by a path. A maximal connected sub graph of $G$ is known as a connected component or simply a component of $G$ thus a disconnected graph as a minimum of two parts. A connected graph of order $m, m \geq 1$ with size $m-1$ and no cycle is called $\boldsymbol{a}$ tree. The shortest path joining between two vertices $v$ and $u$ of the length of $Y$ is called distance $\boldsymbol{d}(\boldsymbol{v}, \boldsymbol{u})$. For one directed circuit in a directed graph, the graph it said to be $\boldsymbol{a}$ cyclic graph and it is alleged to be cyclic if have inside no cycles. An acyclic graph is for $G$ that has more than one component, and then $G$ is said to be a forest. For a graph $G$, if $T=\left(V^{\prime}, E^{\prime}\right)$ is a cyclic subgraph of $G$ such that $V=V$ then $T$ is said to be a spanning forest of $G, T$ is said to be a spanning tree if it has precisely one component.

The issue is for finding the minimium spanning tree for the entire connected weighted graph which is termed as the Minimium Spanning Tree Problem. A pairs $(G, w)$ where $G=(V, E)$ is termed a weighted graph and a weight function for the path is $w: E \rightarrow R$ and it is equal to $\sum_{i=1}^{n} w\left(e_{i}\right) .(G, w)$ is a weighted graph with $G=(V, E)$ if $H=\left(V^{\prime}, E^{\prime}\right)$ is sub graph of $G$ is called Sub graph weight, then weight of $H$ is $w(H)=$ $\sum_{e \in E^{\prime}} w(e)$ od. And weighted graph $(G, w)$ with $G=(V, E)$. The minimum spanning forest problem for $G$ is to find a forest $T=\left(V^{\prime}, E^{\prime}\right)$ that is a spanning sub graph of $G$ that has the smallest possible weight. The distance between two vertices represents the weight of all edges that it takes in this article.

Structure of the over complete Graph $G_{I}[9]$

The objective of image segmentation is for looking out the districts that correspond to the purposeful elements of object distinctive, real objects, shadows and pseudo articles or really finds something interest inside the image needs a few kind of segmentation; The method involves the segmentation of blood vessels to find vessel center line dots (vertices) and their related links (edge), though the last contain convalescent the property of those points. This type of segmentation is additionally referred to as vascular reproductin the utilization of graphs permits for a construction illustration of thing models extricated from image. The formation of the over complete graph is presented with the accompanying focuses:

a) Beginning at the first image $I$ and develop graph $G_{I}$ that vertices $V$ and edges $E$. The crossing point of the veins means the vertices $V_{I}$ in the directed graph, the actualize corresponding to as graph $G_{I}$, that contains the state exactly segmentation of the veasculature and along these lines the edges $E_{I}$ symbolize the vessels between two nods.

b) In front of bifurcations and bent segments, edges turn out to be much more. See Figure 1 [15].

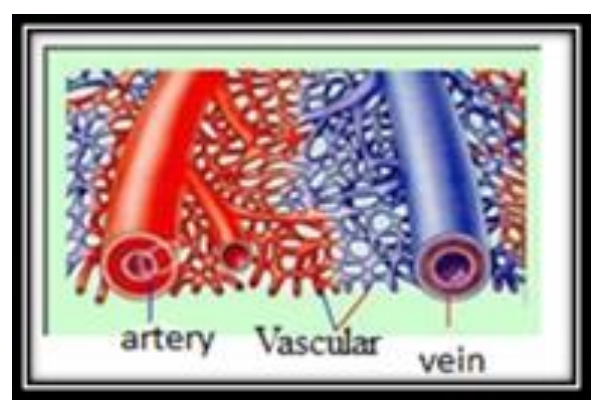

(a)

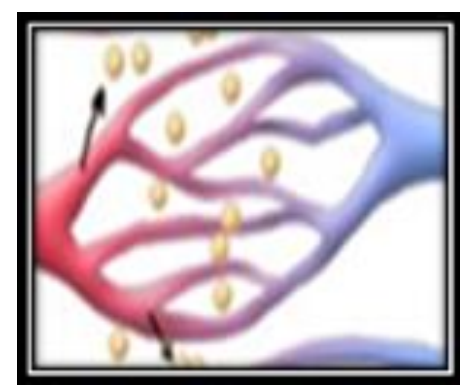

(b)

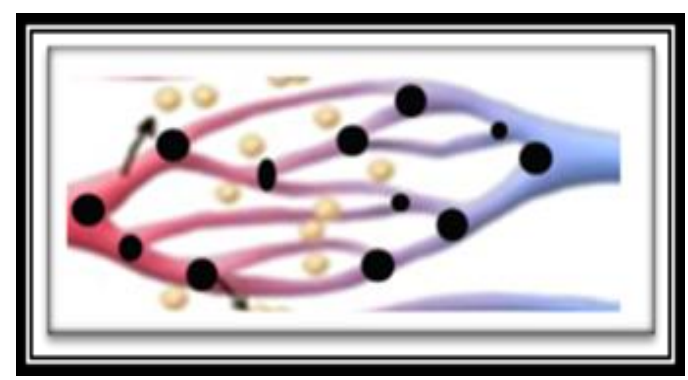

(c)

Figure 1. (a) Medical image segmentation; (The original image); (b) Cutting and zooming (c) structure of the blood-vessel over complete Graph $G_{I}$ 


\subsection{Detection for the place of the vessel clot}

It can find the clotted blood vessels can be found by representing the weight of each edge as the amount of blood in the vessel. If the amount of blood in blood vessel is less than the normal, this mean that the vessel is clotted otherwise it is normal. As given in Figure 2.

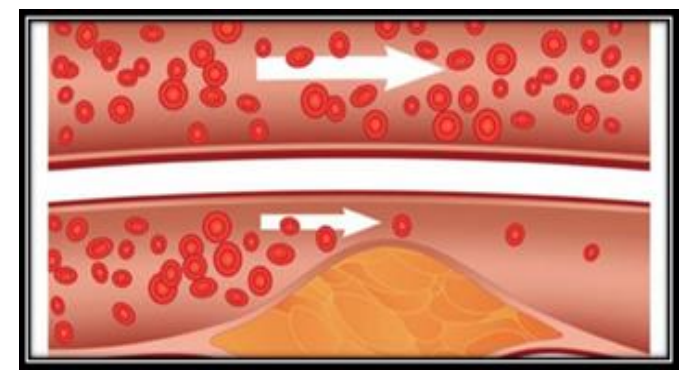

Figure 2. blood vessel clotted

Example 1: To find the clotted blood vessels, let $a$ be the mount of the normal blood and for $\mathrm{i}=1,2,3, \ldots, 17$

$\left\{\right.$ The vessel is not clotted. if $w_{i} \geq a$

The vessel is clotted. if $w_{i}<a$

This is represents as Figure 3.
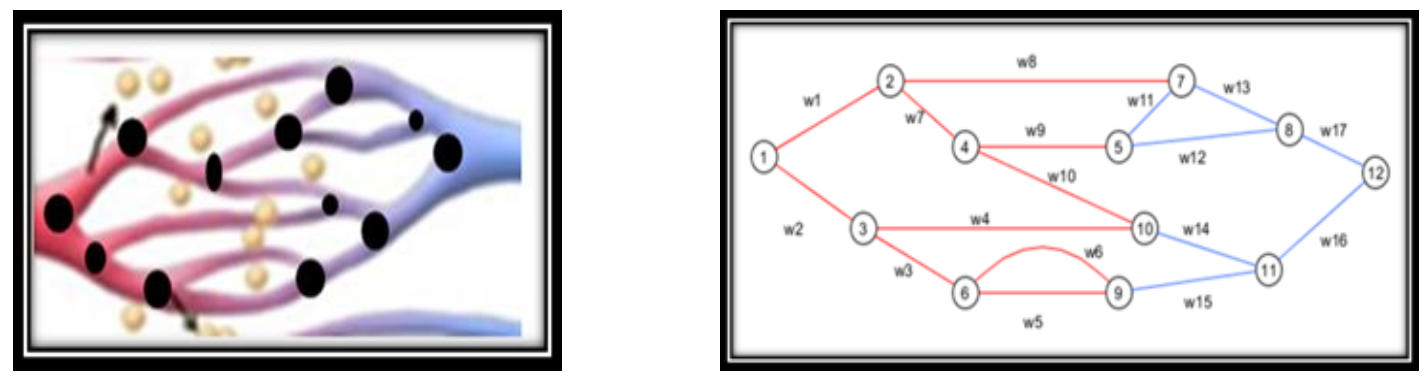

Figure 3. blood vessels

\section{THE SHORTEST PATH IN THE VASCULAR NETWORK}

For a connected subgraph the minimum path could be founded by accomplished through an assortment of network optimization algorithms. The short routes from a source to a sink in the transportation network is founded by the following equation,

$$
f_{i}\left(x_{i}\right)=\min _{\text {all feasible }}\left\{d\left(x_{i-1}, x_{i}\right)+f_{i-1}\left(x_{i-1}\right)\right\}, i=1,2,3
$$

At stage $i$ the function $f_{i}\left(x_{i}\right)$ means the shortest distance to a vertex $x_{i}$, that describes as $d\left(x_{i-1}, x_{i}\right)$ and the distance from vertex $x_{i-1}$ to vertex $x_{i}$; then $f_{i}$ is computed from $f_{i-1}$.

Or in another given by formula:

$$
\left(\begin{array}{c}
\text { shortest distance } \\
\text { to node } x_{i}
\end{array}\right)=\min _{i=1,2,3}\left\{\left(\begin{array}{c}
\text { shortest } \\
\text { distance } \\
\text { to node } i
\end{array}\right)+\left(\begin{array}{c}
\text { distance from } \\
\text { node } i \\
\text { to node } x_{i}
\end{array}\right)\right\}
$$


Also one can take another way to find the short path using linear programming you can see [16-22]. Another method can take to find the shorteset path in graph theory see [23-25].

There are a lot of applications that make the relationship between mathematics and medical application, for more detailes one can found at [26-30].

Example 2: The shortest distance to the clotted vein utilizing the short route problem is down as follow: consider the graph as given in Figure 1(c), set of nodes is $V=\{1,2,3,4,5,6,7,8,9,10,11,12\}$ and the set of edge is $\{1,0.5,1.5,5,4.5,6,2.3,2.5,3.5,6,7,4,3,3.5,2,1.3,8\}$ that represent the weight of blood in the vessel as represents in Figure 4.
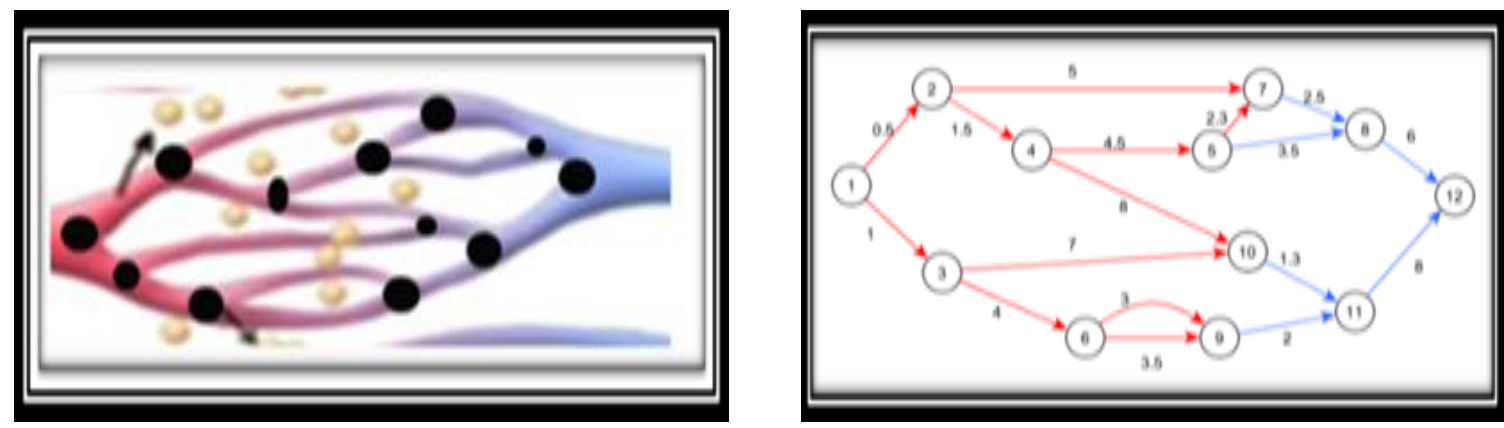

Figure 4. structure of the vessels over the overcomplete graph $G_{I}$

Consider that the vein (edge) which is interfaces of the vertex ten together with the remainder of the veins have a coagulation, until getting the node ten at the shortest distance as follow in Figures 5 and 6 :

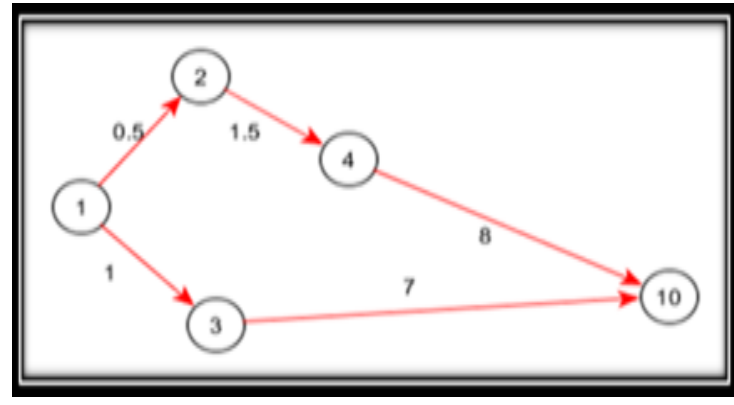

Figure 5. The subgraph over the overcomplete Graph $G_{I}$

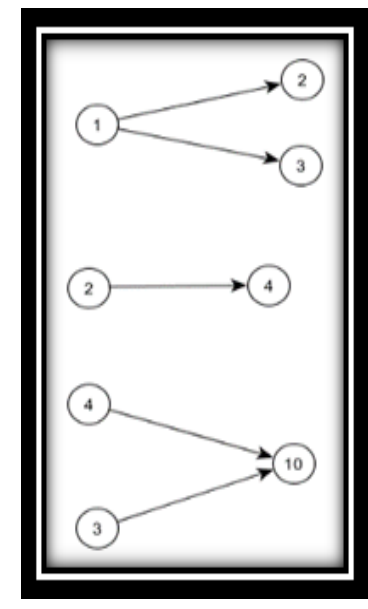

Figure 6. Stages of the shortest routte problem that breaks up into smaller

\section{a) Step1}

The shortest distance from $v_{1}$ towards $v_{2}$ is 0.5 mile.

Shortest distance from $v_{1}$ to $v_{3}$ is 1 mile.

b) Step2

Shortest distance from $v_{2}$ to $v_{4}$ is equal to $0.5+1.5=2$ miles.

c) Step 3

$$
\left(\begin{array}{c}
\text { Shortest distance } \\
\text { to node } 10
\end{array}\right)=\min _{i=3,4}\left\{\left(\begin{array}{c}
\text { shortest } \\
\text { distance } \\
\text { to node } i
\end{array}\right)+\left(\begin{array}{c}
\text { distance from } \\
\text { node I to } \\
\text { node } 10
\end{array}\right)\right\}=
$$




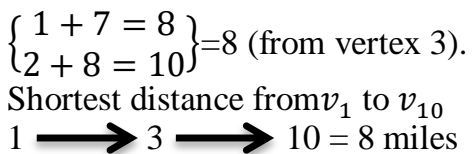

That is the shortest distance is equal to 8 .

\section{CONCLUSION}

As indicated by the significant of human kind and the symptoms of the cutting edge life we utilized mathematics for giving the association of the graph and the vascular network, to arrive the coagulation that damage a vessel using minimum path, algorithms were utilized such that the nods of the graph show up as the vascular ending points and the edges means the vein while the weigh takes the measure of blood. To discover the connection of graph and vascular network, Graph theory is utilized making an answer for the considered problem.

\section{REFERENCES}

[1] R. Abhilasha, "Minimum cost spanning tree using prims algorithm." International journal of advance Research in computer science and management studies, vol. 1, no. 1, 2013.

[2] M.Remper, B. Andres, B. Menze, "The minimum cost connected subgraph problem in medical image analysis," Internat. Conf. on medical image computing and computer-assisted Intervention MICCAI, 2016.

[3] Sanfeliu, et al., "Graph-based representations and techniques for image processing and image analysis," Pattern recognition, vol. 35, no. 3, pp. 639-650, 2002.

[4] S.N.Safuan, et al., "Investigation of white blood cell biomaker model for acute lymphoblastic leukemia detection based on convolutional neural network," Bulletin of Electrical Engineering and Informatics (BEEI), vol. 9, no. 2, pp. 611-618, 2020.

[5] J.S. Duncan, et al, and Nicholas Ayache, "Medical image analysis: Progress over two decades and the challenges ahead," IEEE transactions on pattern analysis and machine intelligence, vol. 22, no. 1, pp. 85-106, 2000.

[6] A.Sanfeliu, et al., "Graph-based representations and techniques for image processing and image analysis," Pattern recognition, vol. 35, no. 3, pp. 639-650, 2002.

[7] E.Türetken, et al.,"Reconstructing curvilinear networks using path classifiers and integer programming", IEEE transactions on pattern analysis and machine intelligence, vol. 38, no. 12, pp. 2515-2530, 2016.

[8] M. Rempfler, et al.,"Reconstructing cerebrovascular networks under local physiological constraints by integer programming", Medical image analysis, vol. 25, no. 1, pp. 86-94, 2015.

[9] D. Robben, et al., "Simultaneous segmentation and anatomical labeling of the cerebral vasculature". Medical image analysis, vol. 32, pp. 201-215, 2016.

[10] C. Payer, et al.,"Automated integer programming based separation of arteries and veins from thoracic CT images". Medical image analysis, vol. 34, pp. 109-122, 2016.

[11] R. Balakrishnan, et al., "Directed Graphs. In A Textbook of Graph Theory". Springer, New York, NY, pp. 37-47, 2012.

[12] M. S. Rahman, "Basic graph theory, Cham," Springer, p. 3, 2017.

[13] G. Chartrand et al., "A first course in graph theory," Courier Corporation, 2013.

[14] S. A. Salman, et al., "The Minimum Cost Connected Subgraph for the Vascular Network," Energy Procedia, vol. 157, 128-134, 2019.

[15] From http://www.moqatel.com/openshare/Behoth/MSehia10/SihiyahMou/Blood/fig22.gif_cvt.htm

[16] N. F. Ibrahim, et al., "Cheap vs healthy: Analyzing McDonald's menu using linear programming," Bulletin of Electrical Engineering and Informatics (BEEI), vol. 9, no. 2, pp. 771-776, 2020.

[17] A.Taha Hamdy, "Operations research: an introduction," Pearson Education India, 2013.

[18] S.S. Rao, "Optimization theory and applications, "John Wiley \& Sons, Inc., 605 Third Ave., New York, Ny 10158, Usa, p. 550, 1983.

[19] E. Türetken, et al., "Reconstructing curvilinear networks using path classifiers and integer programming, " IEEE transactions on pattern analysis and machine intelligence, vol. 38, no. 12, pp. 2515-2530, 2016.

[20] D.P.Williamson, "Lecture 4," ORIE 6300 Mathematical Programming I, 2014.

[21] M.S.Bazaraa, et al., "Linear programming and network flows, "John Wiley \& Sons. 2011.

[22] Shatha, H. Abeer, "The Minimum Cost for the Vascular Network using linear programming based its path graph," Iraqi journal of science, vol. 60, no. 4, pp. 859-867, 2019.

[23] Griffin, "Graph Theory: Penn State Math 485 Lecture Notes," 2017.

[24] Yi, Faliu, and Inkyu Moon, "Image segmentation: A survey of graph-cut methods," In 2012 International Conference on Systems and Informatics (ICSAI2012), pp. 1936-1941. IEEE, 2012.

[25] K.Rvohone, "graph theory, "Tampere University of Technology, 2008.

[26] S.M. Rosli, M.M. Rosli, et al., "A mapping study on blood glucose recommender system for patients with gestational diabetes mellitus," Bulletin of Electrical Engineering and Informatics (BEEI), vol. 8, no. 4, pp.1489-1495, 2019.

[27] Z. Saringat, et al., "Comparative analysis of classification algorithms for chronic kidney disease diagnosis," Bulletin of Electrical Engineering and Informatics (BEEI), vol. 8, no.4, pp. 1496-1501, 2019. 
[28] Bossard,et al., "Supporting reconstruction of the blood vessel network using graph theory: an abstraction method," In 2012 Annual International Conference of the IEEE Engineering in Medicine and Biology Society, pp. 5470-5473. IEEE, 2012.

[29] F. Malmberga, b, J. Lindblad, et al., "A graph-based framework for sub-pixel image segmentation," Theoretical Computer Science, vol. 412, no. 15, pp. 1338-1349, 2011.

[30] R. C. Gonzalez, R. E. Woods, “Digital Image Processing,” Pearson Education, Inc, 2008.

\section{BIOGRAPHIES OF AUTHORS}

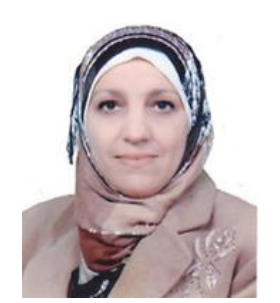

Shatha Salman is Ph.D. degree from Al-Nahrain University in 2005. She has become assistant professor on November 2005. Her Master of Applied Mathematics from University of Technology Iraq (1995). She obtained Bachelor Degree in Applied Mathematics from University of Technology (Iraq) in 1990. Her researches are in fields of Geometric Combintorics, Abstract Algebra, Graph theory, Differential Equations Ordinary and Fractional, Numerical Analysis and Complex Analysis. As well, she has been a member of Iraqi of Scientific committee since 1995. She has served as invited reviewer. She has 40 published articles inside Iraq and some in international journals, 2 published books in Germany. Further info on his scholar.google. https://scholar.google.com/citations?user=_WbhHyQAAAAJ\&hl=en\&authuser=1

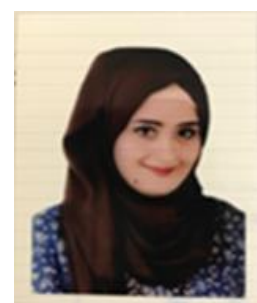

Abeer Hussin Abd-Almeer is a graduate research assistant at University of Baghdad-Departmaent of Mathematics with Master of Applied Mathematics from University of Technology Iraq (2018). She obtained Bachelor Degree in Applied Mathematics from University of Technology (Iraq) in 2016. Her researches are in fields of Geometric Combintorics, Abstract Algebra, Graph theory, She has some published articles inside Iraq and some in international journals. 